\title{
CARACTERÍSTICAS CLÍNICAS DE PACIENTES PEDIÁTRICOS COM CONSTIPAÇÃO CRÔNICA DE ACORDO COM O GRUPO ETÁRIO
}

\author{
Lilian Cristiane da Silva MEDEIROS'1, Mauro Batista de MORAIS², Soraia TAHAN², Érika FUKUSHIMA², \\ Maria Eugênia Farias Almeida MOTTA e Ulysses FAGUNDES-NETO²
}

RESUMO - Racional - Não existem estudos avaliando as características clínicas de constipação de acordo com os grupos etários pediátricos. Objetivo - Avaliar as características da constipação crônica de acordo com o grupo etário. Métodos - Estudo retrospectivo para avaliar informações demográficas e características clínicas de pacientes pediátricos atendidos em ambulatório especializado entre maio de 1995 e dezembro de 2002. Os dados obtidos foram analisados de acordo com o grupo etário (lactentes, pré-escolares, escolares e adolescentes). Resultados - Foram estudados 561 pacientes, predominando o grupo etário de pré-escolares (42,9\%), seguido de escolares (26,9\%), lactentes $(19,1 \%)$ e adolescentes (11,0\%). Não se observou diferença estatisticamente significante entre os gêneros nas quatro faixas etárias. Menos que três evacuações por semana foi observado em maior percentual nos pré-escolares $(65,8 \%)$ e escolares $(59,6 \%)$ do que nos lactentes $(52,4 \%)$ e adolescentes $(43,1 \%)$. Escape fecal foi observado em $75,6 \%$ dos pré-escolares com idade maior do que 48 meses, $68,2 \%$ dos escolares e $76,7 \%$ dos adolescentes. Maior freqüência de escape fecal no gênero masculino $(80,7 \%)$ do que no feminino (50,0\%) foi observada apenas no grupo etário de escolares. Comportamento de retenção $(67,6 \%, 40,7 \%, 27,2 \%)$, medo de defecar $(70,2 \%, 44,2 \%$, $29,7 \%)$ e dor abdominal $(80,8 \%, 69,6 \%, 73,6 \%)$ foram observados, respectivamente, com maior freqüência nos pré-escolares do que nos escolares e adolescentes. Conclusão - Observaram-se diferenças nas características clínicas de pacientes com constipação crônica segundo o grupo etário, no entanto, em todas as faixas etárias observou-se duração prolongada da queixa de constipação e elevada freqüência de complicações como dor abdominal e escape fecal.

DESCRITORES - Constipação intestinal. Distribuição por idade. Pré-escolar. Criança. Adolescente.

\section{INTRODUÇÃO}

Constipação intestinal crônica é um problema freqüente na população pediátrica, estimando-se que seja a principal queixa em 3\% das consultas de pediatria e $25 \%$ das consultas em ambulatórios de gastroenterologia pediátrica ${ }^{(8)}$. Em nosso meio, a prevalência de constipação intestinal varia entre $17,5 \%$ e $36,5 \%$, de acordo com informações obtidas em estudos realizados em unidades básicas de saúde, ambulatórios de pediatria, escolas e na comunidade $^{(1,4,5,11,15,18)}$. Apesar da elevada prevalência de constipação, observa-se pouca valorização desse sintoma por parte dos pais e profissionais de saúde ${ }^{(5)}, \mathrm{o}$ que ocasiona retardo no início do tratamento, favorecendo o surgimento de complicações ${ }^{(10)}$. Por sua vez, existem poucas publicações que descrevem com detalhes as características clínicas de pacientes com constipação crônica atendidos em ambulatórios de gastroenterologia pediátrica $^{(9,10,17)}$. Em nosso conhecimento, não existe nenhum estudo em que tenha sido realizada a comparação das suas características clínicas nas diferentes faixas etárias da população pediátrica.

No presente estudo foi realizada uma análise retrospectiva com o objetivo de avaliar as características clínicas de pacientes com constipação crônica de acordo com o grupo etário.

\section{MÉTODOS}

Neste estudo retrospectivo foram avaliadas as informações coletadas na primeira consulta de 561 pacientes com diagnóstico de constipação crônica

Disciplina de Gastroenterologia Pediátrica, Universidade Federal de São Paulo, Escola Paulista de Medicina - UNIFESP-EPM, São Paulo, SP.

${ }^{1}$ Curso de Pós-graduação em Nutrição, UNIFESP-EPM; '² Disciplina de Gastroenterologia Pediátrica da UNIFESP-EPM; ${ }^{3}$ Departamento Materno-Infantil da Universidade Federal de Pernambuco, Recife, PE

Correspondência: Mauro Batista de Morais - Rua Pedro de Toledo, 441 - 04039-031 - São Paulo, SP. E-mail: mbmorais@osite.com.br 
atendidos consecutivamente no Ambulatório de Motilidade da Disciplina de Gastroenterologia Pediátrica da Universidade Federal de São Paulo - Escola Paulista de Medicina, no período de maio de 1995 a dezembro de 2002. Na primeira consulta deste ambulatório é utilizado um questionário estruturado que aborda informações clínicas obtidas de maneira categorizada. Para o presente estudo, as informações constantes dos prontuários sobre as características do hábito intestinal, idade de início da constipação intestinal, duração da queixa, sinais e sintomas associados e achados do exame físico foram coletadas em um formulário específico. Escape fecal foi caracterizado nos pacientes com idade igual ou maior do que 48 meses. As informações foram transferidas para uma planilha do programa Microsoft Office Excel ${ }^{\mathbb{B}}$ para serem analisadas.

Constipação crônica foi caracterizada pela eliminação de fezes endurecidas com dificuldade ou dor, associadas ou não à ocorrência de comportamento de retenção, escape fecal e aumento do intervalo entre as evacuações ${ }^{(6,13,14)}$. Foram excluídos da avaliação os pacientes com diagnóstico de doença de Hirschsprung, constipação secundária à alergia ao leite de vaca, crianças com encefalopatia crônica ou pacientes com evidências clínicas de constipação secundária a anormalidades anatômicas (estenose anal) ou funcionais (pseudobstrução intestinal) do intestino e outras doenças como doença celíaca e síndromes genéticas. Assim, a casuística foi constituída por pacientes com hipótese diagnóstica de constipação crônica funcional.

Para análise dos dados, os pacientes foram distribuídos de acordo com a faixa etária: lactentes ( 0 a 23,9 meses), pré-escolares (24,0 a 71,9 meses), escolares ( 72 a 120 meses) e adolescentes (121 a 229 meses).
Para análise dos resultados foi utilizado o teste do Qui-quadrado para as variáveis categóricas e a partição do Qui-quadrado para a identificação de diferenças entre as faixas etárias. $\mathrm{O}$ teste de Kruskal-Wallis, complementado por teste de comparações múltiplas, quando necessário, foi utilizado para as variáveis contínuas. Fixou-se em 0,05 ou 5\% o nível de rejeição da hipótese de nulidade. Os cálculos foram realizados com o emprego do programa de estatística SigmaStat ${ }^{\mathbb{}}$ for Windows.

\section{RESULTADOS}

Nesta amostra, constituída por 561 pacientes, ocorreu a seguinte distribuição por faixa etária: pré-escolares $(42,9 \%)$, escolares $(26,9 \%)$, lactentes $(19,1 \%)$ e adolescentes $(11,0 \%)$. Apesar do predomínio de pacientes do sexo masculino (56,5\%, 317/561) e do aumento da proporção masculino:feminino com o aumento da idade, não se observou diferença estatisticamente significante entre os gêneros nas quatro faixas etárias (Tabela 1). Nessa tabela observa-se, também, que o intervalo entre o início da constipação e a primeira consulta no ambulatório especializado foi maior nos adolescentes e escolares, em comparação com os pré-escolares e lactentes. Por sua vez, este parâmetro foi maior nos pré-escolares do que nos lactentes. Assim, pode-se dizer que quanto maior a idade, maior a duração da queixa de constipação por ocasião da primeira consulta.

Na Tabela 2 pode ser observado que o número de evacuações inferior a três vezes por semana ocorreu em menor proporção nos lactentes e adolescentes. A ocorrência de dor durante a evacuação foi maior nos lactentes, lembrando que essa informação foi obtida com base na percepção dos pais ou responsáveis. A proporção de pacientes com eliminação de fezes endurecidas e ressecadas não diferiu entre os grupos etários.

TABELA 1. Distribuição dos pacientes com constipação crônica de acordo com o grupo etário

\begin{tabular}{|c|c|c|c|c|c|}
\hline & Lactente $(\mathrm{n}=107)$ & Pré-escolar $(\mathrm{n}=241)$ & $\operatorname{Escolar}(\mathrm{n}=151)$ & Adolescente $(n=62)$ & $P$ \\
\hline Sexo (masculino/feminino) ${ }^{*}$ & $52 / 55(0,9: 1,0)$ & $135 / 106(1,3: 1,0)$ & $90 / 61(1,5: 1,0)$ & $40 / 22(1,8: 1,0)$ & 0,176 \\
\hline Idade, em meses, na primeira consulta ${ }^{\dagger}$ & $14,0(9,0-19,0)$ & $42,0(31,7-53,2)$ & $95,0(82,0-106,0)$ & $135,5(127,0-142,0)$ & \\
\hline Idade, em meses, de início da constipação ${ }^{\dagger \neq}$ & $3,0(0,0-8,0)$ & $12,0(2,0-29,5)$ & $47,0(6,0-73,0)$ & $71,0(35,2-109,2)$ & $<0,001$ \\
\hline $\begin{array}{l}\text { Intervalo, em meses, entre o início da constipação } \\
\text { e a primeira consulta }{ }^{\dagger \ddagger}\end{array}$ & $8,0(4,0-13,0)$ & $24,0(12,0-36,0)$ & $55,0(24,0-77,0)$ & $68,0(24,0-114,0)$ & $<0,001$ \\
\hline Início da constipação no $1^{\circ}$ ano de vida"s॥ ${ }^{*} \|$ & $90,2 \%(92 / 102)$ & $52,6 \%(120 / 228)$ & $30,1 \%(40 / 133)$ & $15,7 \%(9 / 57)$ & $<0,001$ \\
\hline
\end{tabular}

* Teste do Qui-quadrado

$\dagger$ Mediana e percentis $25-75$ entre parênteses

† Teste de Kruskal-Wallis complementado pelo teste de comparações múltiplas de Dunn:

Idade de início da constipação: adolescente > lactente, pré-escolar e escolar; escolar > lactente e pré-escolar; pré-escolar > lactente

Intervalo entre o início da constipação e a primeira consulta: adolescente > lactente e pré-escolar; adolescente não difere significantemente do escolar; escolar > lactente e préescolar; pré-escolar > lactente.

§ Partição do Qui-quadrado: lactente > pré-escolar > escolar > adolescente.

TABELA 2. Características do hábito intestinal dos pacientes com constipação de acordo com o grupo etário

\begin{tabular}{|c|c|c|c|c|c|}
\hline & Lactente & Pré-escolar & Escolar & Adolescente & $P$ \\
\hline$<3$ evacuações por semana ${ }^{* \dagger}$ & $52,4 \%(54 / 103)$ & $65,8 \%(152 / 231)$ & $59,6 \%(84 / 141)$ & $43,1 \%(25 / 58)$ & $<0,01$ \\
\hline Dificuldade para defecar ${ }^{* \neq}$ & $94,1 \%(96 / 102)$ & $89,3 \%(201 / 225)$ & $86,4 \%(121 / 140)$ & $75,4 \%(43 / 57)$ & $<0,01$ \\
\hline Dor durante defecação"§ & $89,6 \%(86 / 96)$ & $79,0 \%(177 / 224)$ & $65,0 \%(93 / 143)$ & $58,6 \%(34 / 58)$ & $<0,001$ \\
\hline Fezes endurecidas e ressecadas* & $84,3 \%(86 / 102)$ & $87,0 \%(201 / 231)$ & $81,0 \%(115 / 142)$ & $84,2 \%(48 / 57)$ & 0,481 \\
\hline
\end{tabular}

Percentagens calculadas de acordo com o total de informações disponíveis

*Teste do Qui-quadrado

Partição do Qui-quadrado:

$\dagger<3$ evacuaçōes/semana: adolescente < lactente, pré-escolar e escolar; lactente< pré-escolar e escolar

₹ Dificuldade para defecar: adolescente < lactente, pré-escolar e escolar
$\S$ Dor durante a defecação: lactente e pré-escolar > escolar e adolescente; lactente > pré-escolar 
TABELA 3. Freqüência de sinais e sintomas associados à constipação crônica funcional de acordo com o grupo etário

\begin{tabular}{|c|c|c|c|c|}
\hline Sinais e sintomas & Pré-escolar & Escolar & Adolescente & $P$ \\
\hline Escape fecal ${ }^{*}$ & $75,6 \%(62 / 84)$ & $68,2 \%(101 / 148)$ & $76,7 \%(46 / 60)$ & 0,411 \\
\hline \multicolumn{5}{|c|}{ Freqüência de escape segundo o gênero ${ }^{\dagger}$} \\
\hline Masculino & $76,6 \%(36 / 47)$ & $80,7 \%(71 / 88)$ & $74,3 \%(29 / 39)$ & \\
\hline Feminino & $70,3 \%(26 / 37)$ & $50,0 \%(30 / 60)$ & $81,0 \%(17 / 21)$ & \\
\hline Duração escape/meses ${ }^{\ddagger \S}$ & $12,0(2,8-13,3)$ & $24,0(9,0-48,0)$ & $36,0(12,0-60,0)$ & $<0,001$ \\
\hline Freqüência escape $e^{\ddagger \|}$ & $4,0(2,0-14,0)$ & $7,0(3,0-14,0)$ & $7,0(3,2-21,0)$ & 0,104 \\
\hline \multicolumn{5}{|l|}{ Número de vezes/semana } \\
\hline Dor abdominal ${ }^{\pi}$ & $80,8 \%(160 / 198)$ & $69,6 \%(87 / 125)$ & $73,6 \%(39 / 53)$ & $<0,001$ \\
\hline Manobra de retenção" & $67,6 \%(123 / 182)$ & $40,7 \%(42 / 103)$ & $27,2 \%(12 / 44)$ & $<0,001$ \\
\hline Medo de defecar" & $70,2 \%(139 / 198)$ & $44,2 \%(54 / 122)$ & $29,7 \%(14 / 47)$ & $<0,001$ \\
\hline Medo de se sentar no sanitário & $45,2 \%(76 / 168)$ & $26,2 \%(26 / 99)$ & $10,0 \%(4 / 40)$ & $<0,001$ \\
\hline
\end{tabular}

Percentagens calculadas de acordo com o total de informações disponíveis

Para as variáveis presença de escape fecal, escape fecal de acordo com o gênero, duração e freqüência do escape foram considerados apenas os pré-escolares com idade maior ou igual a 48 meses (n $=84$ ).

* Presença de escape fecal - Partição do Qui-quadrado: pré-escolar < escolar e adolescente

$\dagger$ Frequêencia de escape segundo o gênero: pré-escolar, $P=0,685$; escolar, $P=0,0001$; adolescente $P=0,75$

‡ Kruskal-Wallis complementado pelo teste de comparações múltiplas de Dunn

§. Duração escape/meses: préescolar < escolar e adolescente $(P<0,05)$

TABELA 4. Características do exame físico nos pacientes com constipação crônica funcional de acordo com o grupo etário

\begin{tabular}{|c|c|c|c|c|c|}
\hline & Lactente & Pré-escolar & Escolar & Adolescente & $P$ \\
\hline Distensão abdominal & $12,6 \%(13 / 103)$ & $15,1 \%(30 / 229)$ & $11,1 \%(16 / 144)$ & $10,7 \%(6 / 56)$ & 0,927 \\
\hline Massa fecal palpável* & $26,5 \%(26 / 98)$ & $45,7 \%(101 / 221)$ & $47,1 \%(65 / 138)$ & $45,5 \%(25 / 55)$ & $<0,01$ \\
\hline Fissura anal & $8,9 \%(8 / 90)$ & $5,5 \%(11 / 201)$ & $3,2 \%(4 / 125)$ & $7,1 \%(3 / 41)$ & 0,33 \\
\hline Plicoma & $14,6 \%(13 / 89)$ & $18,1 \%(36 / 199)$ & $13,3 \%(16 / 120)$ & $17,0 \%(7 / 41)$ & 0,69 \\
\hline Fezes endurecidas na ampola retal $^{\dagger}$ & $46,1 \%(30 / 65)$ & $55,5 \%(85 / 153)$ & $37,3 \%(37 / 99)$ & $33,3 \%(14 / 42)$ & $<0,001$ \\
\hline
\end{tabular}

Percentagens calculadas de acordo com o total de informações disponíveis

Partiçāo do Qui-quadrado:

A comparação entre as proporções de ocorrência de escape fecal entre pré-escolares com mais de 48 meses, escolares e adolescentes não revelou diferença estatisticamente significante (Tabela 3 ). $\mathrm{Na}$ avaliação da freqüência de escape fecal de acordo com o gênero, verificou-se nos escolares, predomínio estatisticamente significante no sexo masculino $(P=0,001)$. Nos adolescentes a freqüência de escape fecal foi superior no sexo feminino, mas o estudo estatístico não revelou diferença significante. Em relação aos escolares e adolescentes, os pré-escolares apresentaram maior proporção de relatos de dor abdominal, manobras de retenção, medo de defecar e medo de se sentar no vaso sanitário (Tabela 3). Não se observou diferença estatisticamente significante entre essas variáveis entre adolescentes e escolares, apesar das proporções serem menores nos adolescentes (Tabela 3).

Ao exame físico, não se observaram diferenças estatisticamente significantes na ocorrência de distensão abdominal, fissura anal e plicoma. Massa fecal palpável foi detectada em menor proporção nos lactentes e a presença de fezes endurecidas na ampola retal, nos pacientes submetidos ao toque retal, foi maior nos pré-escolares, quando comparados aos demais grupos (Tabela 4).

\section{DISCUSSÃO}

Constipação é um sintoma freqüente na faixa etária pediátrica. Estudos realizados no Brasil mostram que sua prevalência é elevada na comunidade ${ }^{(1,4,5,11,15,18)}$, no entanto, existem evidências de que as características clínicas desses casos identificados

na comunidade apresentam menor gravidade do que aqueles atendidos em ambulatórios de gastroenterologia pediátrica ${ }^{(2)}$. A descrição das características clínicas de pacientes com constipação crônica atendidos em serviços de referência foi motivo de alguns estudos publicados, no entanto, segundo os presentes autores, suas particularidades de acordo com todas as diferentes faixas etárias da pediatria não foram avaliadas em nenhum deles. Este estudo retrospectivo analisa as características clínicas de pacientes atendidos com constipação crônica em um ambulatório de gastroenterologia pediátrica, distribuídas segundo os quatro grupos etários pediátricos: lactente, préescolar, escolar e adolescente.

Vale ressaltar que os dados relativos à duração da constipação por ocasião da primeira consulta, assim como a idade de seu início e a duração do escape fecal, devem ser interpretados levando-se em conta o grupo etário, ou seja, quanto menor a idade, teoricamente, esses valores tendem a ser menores. Quanto ao escape fecal, considerou-se sua presença apenas após os 48 meses de idade. Na prática, escape fecal pode ser caracterizado após a obtenção do controle esfincteriano, mesmo antes dos 48 meses $^{(13)}$, no entanto, as informações coletadas nos prontuários não permitiram relacioná-lo com controle esfincteriano. Por outro lado, escape fecal constava de $71(52,2 \%)$ dos 136 prontuários de pré-escolares com idade inferior a 48 meses (dados não considerados nos Resultados).

Nos 107 lactentes estudados não se observou predomínio no gênero masculino, como ocorre nas faixas etárias mais 
elevadas. Nessa faixa etária, as medianas da idade de início da constipação e da primeira consulta foram, respectivamente, 3 e 14 meses. A duração mediana da constipação por ocasião da primeira consulta foi de 8 meses, inferior a outras faixas de idade, sendo a diferença estatisticamente significante. As proporções de lactentes com massa abdominal palpável $(26,5 \%)$ e freqüência de evacuações inferior a três por semana $(52,4 \%)$ também foram inferiores às observadas nos grupos com maior idade. Este último dado contrasta com o observado em 69 lactentes com constipação identificados em estudo realizado em unidades básicas do município do Embu, SP, nos quais apenas $5,8 \%$ evacuavam menos que três vezes por semana ${ }^{(1)}$. Por sua vez, LOENING-BAUCKE(7), estudando retrospectivamente 132 lactentes (52\% do sexo masculino) com constipação crônica, atendidos em ambulatório terciário de Iowa, nos Estados Unidos, observou que a idade média dos pacientes na primeira consulta era igual a 13,7 meses, a duração média da constipação igual a 6,7 meses, presença de massa abdominal palpável em $13 \%$ e freqüência de evacuação menor do que 3,5 vezes por semana em cerca de $35 \%$ dos lactentes. Esses dados são muito semelhantes aos obtidos no presente estudo, revelando gravidade similar da constipação nesses dois serviços especializados.

Nos pré-escolares, escolares e adolescentes nota-se aumento progressivo na proporção de constipação no gênero masculino, atingindo a proporção de 1,8 masculinos para cada feminino (64,5\% dos casos em adolescentes masculinos). Quanto à percentagem de casos com início da constipação no $1^{\circ}$ ano de vida, observaram-se valores decrescentes que passaram de $52,6 \%$ nos pré-escolares, para $30,1 \%$ nos escolares e $15,7 \%$ nos adolescentes. Estudo realizado em Botucatu, SP, por MAFFEI et al. ${ }^{(10)}$, incluiu 163 pacientes com idade entre 2 e 146 meses (51,5\% do sexo masculino) atendidos com constipação crônica em ambulatório especializado, entre 1981 e 1989. Observouse grande intervalo entre o início da constipação e a primeira consulta (mediana $=38$ meses), mediana da idade de início da constipação no $3^{\circ}$ mês de vida, menos que três evacuações por semana em $59,5 \%$, escape fecal em $56,0 \%$ dos meninos e em $34,2 \%$ das meninas. Nesse mesmo estudo, constatou-se a presença de enurese e infecção urinária atual ou pregressa em cerca de $10 \%$ a $20 \%$ dos pacientes com constipação crônica, manifestações que não foram avaliadas no presente levantamento retrospectivo. Em ambulatório especializado de Iowa, EUA, em 174 crianças com idade inferior a 4 anos e 215 com idade superior a 4, observouse, respectivamente, início da constipação no $1^{\circ}$ ano de vida em $84 \%$ e $49 \%$, comportamento de retenção em $97 \%$ e $71 \%$, massa abdominal palpável em $42 \%$ e $42 \%$ e, finalmente, escape fecal em $90,5 \%$ dos maiores de 4 anos $^{(9)}$. Esses dados reafirmam que parcela expressiva dos pacientes atendidos em serviços especializados apresenta constipação desde o $1^{\circ}$ ano de vida, antes do treinamento esfincteriano, muito valorizado no passado como fator desencadeante da constipação. Revisão da literatura ${ }^{(17)}$ baseada em 13 artigos publicados, aponta que a constipação na criança provoca redução da freqüência de evacuações em $80 \%$ a $100 \%$ dos pacientes, incontinência fecal (escape fecal) em 35\% a
$96 \%$ e massa fecal abdominal palpável em 30\% a 71\% dos casos, demonstrando que as diferentes casuísticas podem apresentar grandes variabilidades nas características clínicas decorrentes da constipação crônica. No entanto, de forma geral, é possível afirmar que os pacientes incluídos neste estudo apresentam características clínicas similares às encontradas nos serviços especializados do Brasil e de outros países, ou seja, longa duração do sintoma de constipação, presença de complicações por ocasião da procura pelo serviço especializado, especialmente escape fecal e dor abdominal e intervalos prolongados entre as evacuações.

No grupo adolescente, observa-se que o início da constipação no $1^{\circ}$ ano de vida ocorre com menor freqüência, bem como comportamento de retenção, medo de evacuar e de sentar no vaso sanitário. Persiste, no entanto, elevada prevalência de escape fecal que apareceu de forma similar em ambos os sexos. Dos estudos mencionados acima ${ }^{(9,17)}$, nenhum diferenciou as características clínicas da constipação na adolescência. ZASLAVSKY et al. ${ }^{(19)}$ estudaram 48 adolescentes com constipação caracterizada por freqüência de evacuação menor do que 3 vezes por semana por pelo menos 1 ano. Observaram predomínio do sexo feminino na proporção de 2,7:1,0, presença de escape fecal em $23 \%$, fecaloma em $64 \%$ e massa fecal palpável em $39 \%$. Os pacientes da presente série apresentaram maior freqüência de escape fecal $(76,7 \%)$ e menor percentual de freqüência de evacuações inferior a três por semana $(43,1 \%)$, principal critério de inclusão do mencionado estudo. Essas diferenças indicam a importância da padronização das definições dos distúrbios da evacuação na população pediátrica ${ }^{(3,6,12,16)}$.

Características comuns em todas as faixas etárias foram o grande intervalo entre o início da constipação e a primeira consulta em serviço especializado, ausência de aumento no intervalo das evacuações em parcela expressiva dos pacientes e elevada ocorrência de escape fecal e dor abdominal como complicações da constipação crônica funcional.

\section{CONCLUSÕES}

Foram observadas diferenças nas características clínicas de pacientes com constipação crônica de acordo com o grupo etário, no entanto, em todas as faixas etárias encontrou-se duração prolongada da queixa de constipação e elevada freqüência de complicações, especialmente escape fecal e dor abdominal crônica. A longa duração da queixa antes do atendimento sugere que, em todas as faixas etárias, deve-se realizar busca ativa de constipação em todas as consultas, no sentido de se adotar medidas terapêuticas antes do surgimento das complicações freqüentemente observadas nos pacientes atendidos em ambulatórios especializados.

\section{AGRADECIMENTOS}

À Milena Catani, Soraya Goshima e Valéria Cristina Antunes Lisboa pela colaboração na assistência aos pacientes e/ou levantamento de dados e ao Prof. Dr. Neil Ferreira Novo e à Profa. Dra. Yara Juliano pela revisão da análise estatística. 
Medeiros LCS, Morais MB, Tahan S., Fukushima E, Motta MEFA, Fagundes-Neto U. Clinical characteristics of pediatric patients with chronic constipation according to age group. Arq Gastroenterol. 2007;44(4):340-4.

ABSTRACT - Background - There was no study evaluating clinical characteristics of constipation according pediatric age groups. Aim - To evaluate the clinical characteristics of patients with chronic constipation according to age group. Methods - This retrospective study evaluated the demographic data and clinical characteristics of pediatric patients with chronic constipation who had been admitted to an outpatient clinic between May 1995 and December 2000 . Data was analyzed according to the followings age groups: infants, pre-school, school age and adolescent. Results - Five hundred and sixty one patients were included in the study. The percentage of patients according to age groups were: infants (19.1\%), pre-school (42.9\%), school age (26.9\%), and adolescents $(11.0 \%)$. There was no statistical gender difference among the four age groups. Less than three bowel movements per week were observed more frequently in pre-school $(65.8 \%)$ and school age (59.6\%) than in infants $(52.4 \%)$ and adolescents $(43.1 \%)$. Fecal escape ("soiling") was found in $75.6 \%$ of the preschool patients aged more than 48 months, in $68.2 \%$ of the school age and in $76.7 \%$ of the adolescents. Soiling was more frequent in boys ( $80.7 \%$ ) than in girls $(50.0 \%)$ only in school age children. Retentive posture $(67.6 \%, 40.7 \%, 27.2 \%)$, fear for defecation $(70.2 \%, 44.2 \%, 29.7 \%)$ and abdominal pain $(80.8 \%$, $69.6 \%, 73.6 \%$ ) were observed, respectively, more frequently in pre-school than in school age and adolescents. Conclusion - There were differences in clinical characteristics according to age group. However, prolonged duration of constipation and an elevated number of complications were observed in all age groups, especially fecal soiling and abdominal pain.

HEADINGS - Constipation. Age distribution. Child, preschool. Child. Adolescent.

\section{REFERÊNCIAS}

1. Aguirre AN, Vítolo MR, Puccini RF, Morais MB. Constipação em lactentes: influência do tipo de aleitamento e da ingestão de fibra alimentar. J Pediatr (Rio J). 2002;78:202-8.

2. Aquino MD, Silva GS, Oliveira JN, Tahan S, Medeiros LCS, Fagundes-Neto U, Morais MB. Constipação em adolescentes atendidos em ambulatório especializado e em adolescentes identificados em escolas. Anais do XII Congresso Brasileiro de Gastroenterologia Pediátrica - II Congresso Brasileiro de Hepatologia Pediátrica. Gramado, Rio Grande do Sul; 2005.

3. Benninga M, Candy DC, Catto-Smith AG, Clayden G, Loening-Baucke V, Di Lorenzo C, Nurko S, Staiano A. The Paris Consensus on Childhood Constipation Terminology (PACCT) group. J Pediatr Gastroenterol Nutr. 2005;40:273-5.

4. de Araújo Sant'Anna AM, Calçado AC. Constipation in school-aged children at public schools in Rio de Janeiro, Brazil. J Pediatr Gastroenterol Nutr. 1999;29:190-3.

5. Del Ciampo IR, Galvão LC, Del Ciampo LA, Fernandes MI. Prevalência de constipação intestinal crônica em crianças atendidas em unidade básica de saúde. J Pediatr (Rio J). 2002;78:497-502.

6. Hyams J, Colletti R, Faure C, Gabriel-Martinez E, Maffei HV, Morais MB, Hock QS, Vandenplas Y. Functional gastrointestinal disorders: Working Group Report of the First World Congress of Pediatric Gastroenterology, Hepatology and Nutrition. J Pediatr Gastroenterol Nutr. 2002;35 (Suppl 2):s110-s7.

7. Loening-Baucke V. Chronic constipation in children. Gastroenterology. 1993;105:1557-64.

8. Loening-Baucke V. Encopresis and soiling. Pediatr Clin North Am. 1996;43:279-98.

9. Loening-Baucke V. Prevalence, symptoms and outcome of constipation in infants and toddlers. J Pediatr. 2005;146:359-63.
10. Maffei HV, Moreira FL, Kissimoto M, Chaves SM, Faro S, Aleixo AM. História clínica e alimentar de crianças atendidas em ambulatório de gastroenterologia pediátrica com constipação intestinal crônica funcional e suas possíveis complicações. J Pediatr (Rio J). 1994;70:280-6.

11. Maffei HV, Moreira FL, Oliveira WM, Sanini V. Prevalência de constipação intestinal em escolares do ciclo básico. J Pediatr (Rio J). 1997;73:340-4.

12. Maffei HVL, Morais MB. Defining constipation in childhood and adolescence: from Rome, via Boston, to Paris and ...? J Pediatr Gastroenterol Nutr. 2005;41:485-6.

13. Morais MB, Vítolo MR, Aguirre AN, Fagundes-Neto U. Measurement of low dietary fiber intake as a risk factor for chronic constipation in children. J Pediatr Gastroentero Nutr. 1999;29:132-5.

14. Morais MB, Maffei HV. Constipação Intestinal. J Pediatr (Rio J). 2000;76 Suppl 1:s147-s56

15. Motta MEFA, Silva GAP. Constipação intestinal crônica funcional na infância: diagnóstico e prevalência em uma comunidade de baixa renda. J Pediatr. (Rio J). 1998;74:451-4.

16. Rasquin-Weber A, Hyman PE, Cucchiara S, Fleisher DR, Hyams JS, Milla PJ, Staiano A. Childhood functional gastrointestinal disorders. Gut. 1999;45 (Suppl 2):II60-8.

17. van der Plas RN. Management of defecation problems in children. In: van der Plas RN. Clinical management and treatment options in children with defecations disorders. Amsterdam: Academic Medical Center; 1998. p.13-147.

18. Zaslavsky C, Ávila EL, Araújo MA, Pontes MR, Lima NE. Constipação intestinal da Infância. Um estudo de prevalência. Rev AMRIGS. 1988;32:100-2.

19. Zaslavsky C, De Barros SG, Gruber AC, MacIel AC, Da Silveira TR. Chronic functional constipation in adolescents: clinical findings and motility studies. J Adolesc Health. 2004;34:517-22.

Recebido em 10/7/2006 Aprovado em 2/2/2007 